\title{
A Comparison of Time, Frequency and ICA Based Features and Five Classifiers for Wrist Movement Classification in EEG Signals
}

\author{
I. Navarro, F. Sepulveda (Member IEEE), B. Hubais \\ Department of Computer Science, University of Essex, Colchester, UK
}

\begin{abstract}
This study presents a comparison of two methods to extract features for the classification of wrist movements (flexion, extension, pronation, supination). For the first method, a set of $\mathbf{1 6 0}$ features was extracted from the filtered time and frequency domain EEG data and its alpha, beta, and theta bands. For the second method, a set of 40 features per movement type was extracted from the ICAcalculated source signals. The value of the Davies-Bouldin cluster separation index for each feature was used for selecting the best five features from each set so as to avoid the subjective selection or rejection of any of the features. Finally, five different kinds of classifiers were chosen to obtain classification error rates with which to compare both techniques.

The results showed the advantage of using ICA source signals for wrist movement classification purposes, at least as compared to the simple time and frequency domain features. Left and right movements were correctly identified with accuracies ranging from $70 \%$ to $96 \%$. However, the methodology presented here did not succeed in distinguishing the subclasses (e.g., flexion versus extension) with accuracy above $70 \%$. This suggests that additional work is needed to explore different features as well as classifiers.
\end{abstract}

Keywords-Brain-Computer Interface, ICA, Feature Selection, EEG, Movement-Related Potentials

\section{INTRODUCTION}

The successful implementation of an EEG-based BrainComputer Interface (BCI) holds enormous promise for a wide variety of subjects suffering from different types of disorders (e.g., spinal cord injury, stroke, etc.) that affect their ability to perform most daily-life tasks without external help (see [1] for an extensive review on this matter). A fundamental decision that must be taken in the process of designing such a BCI is whether to base the system on the EEG activity in the brain areas normally assigned to the production of those output commands (e.g., sensori-motor cortex). In the present work, which is a continuation of [2], we have chosen to focus on analyzing motor related potentials in all cortical areas, regardless of their expected information content. This approach does not require extensive training and allows us to explore other potential areas that have been ignored in BCI research thus far.

EEG data are always contaminated by artifacts, noise, unwanted spontaneous EEG activity, etc. In addition to this, the skull acts as a spatial low-pass band filter, eliminating much of the information generated intracortically. These factors make it very difficult to obtain a reliable representation of the underlying cortical activity. Hence, the question of how to go about choosing appropriate features to

Address for correspondence: F. Sepulveda, Computer Science, Wivenhoe Park, Colchester - Essex CO4 3SQ, U.K. E-mail: fsepulv@essex.ac.uk represent the EEG data obtained in BCI experiments has resulted in an enormous amount of literature [3] [4] [5]. Time grand averaging, frequency component selection, and spatial pattern analysis have all been tested with various degrees of success. Recently, the independent component analysis (ICA) technique has received an increasing amount of attention [6] [7]. ICA is a statistical methodology that performs blind separation of the sources to obtain statistically-independent components. It is expected that using these components will improve the classifiers' performance.

In the present work, we study the possibility of distinguishing executed and imaginary movements of the left and right wrists in healthy subjects. These movements have been selected for their relevance in future BCI control of hand prostheses. The purpose of the present paper is to compare how simple frequency and time-based features along several frequency bands fare against similar ICA features in helping to identify these wrist movements. This study has been performed in a systematic manner, making use of as few assumptions in the underlying physiological characteristics as possible. The motivation for this approach is to allow the discovery of additional useful trends in the EEG data by avoiding some of the location-related bias in previous research. Finally, the comparative study of the characteristics of the executed and imaginary experiments will be useful in assessing the feasibility of using this approach with locked-in patients.

\section{Methodology}

\section{A. Experimental Procedure}

EEG data were recorded from four healthy male subjects. The cortical patterns were measured with an electrode cap equipped with $21 \mathrm{Ag} / \mathrm{AgCl}$ electrodes in a $20 / 10$ set-up. The data were sampled at a rate of $256 \mathrm{~Hz}$.

For each subject, three 5-minutes experiments were run for each of the following conditions: 1) left-hand executed wrist movements, 2) right-hand executed wrist movement, 3) left-hand imaginary wrist movements, and 4) right-hand imaginary wrist movements.

During each of these experiments, the subjects were instructed to perform one of four wrist movements: a) flex, b) extend, c) pronate, or d) supinate. The commands were shown on a computer screen placed one meter away from the subject. The subjects were given $2 \mathrm{~s}$ to perform the movement after which a return to resting position command was issued. The subjects were given $2 \mathrm{~s}$ before being given an additional command. The exact sequence of wrist movements was randomized. To avoid mental fatigue 
effects, 10-minute breaks were taken after every four 5minute runs.

\section{B. Feature Extraction}

The entire data set was low-pass filtered at $40 \mathrm{~Hz}$ using a Kaiser-window 93rd-order FIR filter in order to clean out unwanted $50 \mathrm{~Hz}$ noise. The epochs corresponding to the EEG data obtained during the previous experiments were then extracted for the two seconds following the appearance of each movement command on screen. Feature extraction task was performed with the help of the EEGLAB utility [8].

Frequency Band Features. The following time-based features were from extracted from the original EEG data: (1) $50 \mathrm{~ms}$ window averages of the rectified EEG amplitude, (2) dominant frequency, (3) time-domain variance over the $2 \mathrm{~s}$ response, and (4) total power over the $2 \mathrm{~s}$ response (using Welch's method).

The original EEG data was then filtered to obtain three additional data sets in which the alpha $(8-13 \mathrm{~Hz})$, beta $(14-$ $30 \mathrm{~Hz})$, and theta $(4-7 \mathrm{~Hz})$ bands were emphasized. The same set of features was extracted from these three additional sets. See Table I for an overall view of the selected features.

TABLE I

TIME-BASED FEATURE NUMBERING AND DESCRPTION

\begin{tabular}{|c|c|}
\hline Feature No. & Description \\
\hline $1-37$ & 50 ms window averages \\
\hline 38 & Dominant frequency \\
\hline 39 & Time-domain variance \\
\hline 40 & Total power \\
\hline $41-77$ & Alpha band 50 ms window averages \\
\hline 78 & Alpha band dominant frequency \\
\hline 79 & Alpha band time-domain variance \\
\hline 80 & Alpha band total power \\
\hline $81-117$ & Beta band 50 ms window averages \\
\hline 118 & Beta band dominant frequency \\
\hline 119 & Beta band time-domain variance \\
\hline 120 & Beta band total power \\
\hline $121-157$ & Theta band 50 ms window averages \\
\hline 158 & Theta band dominant frequency \\
\hline 159 & Theta band time-domain variance \\
\hline 160 & Theta band total power \\
\hline
\end{tabular}

ICA Component Features. ICA is a statistical method whose goal is to find a linear representation of non-Gaussian data so that the components are statistically independent [9]. It is assumed that at time instant $\mathrm{k}$ the observed $\mathrm{n}$-dimensional data vector $\mathrm{x}(\mathrm{k})$ is given by

$$
x(k)=\sum_{i=1}^{n} a_{i} s_{i}=A s(k)
$$

where the $s_{i}$ are the source signals and the $a_{i}$ form the mixing matrix $A$. The source signals are assumed to be stationary and independent. See [7] to review the applicability of these assumptions to the physical processes involved in the production of EEG signals. The goal of the ICA algorithm is to find the mixing matrix $A$ as well as the set of signal sources $s_{i}$. This is achieved by first choosing an appropriate measure of independence or nongaussianity (such as joint entropy, negentropy or kurtosis) and applying a recursive algorithm that maximizes this value for the source signals. The details of the Infomax ICA algorithm used in the present work can be found in [10]. The solution provided by the ICA algorithm takes the form

$$
\tilde{s}(k)=B x(k)
$$

where the $B$ matrix is called the separating matrix and the $\tilde{s}(k)$ are the ICA estimation of the source signals.

Quite often previous researchers have used ICA to reject data artifacts or handpick certain components of physiological interest. However, there are certain theoretical and practical problems associated with this approach. The ICA algorithm does not solve the inverse EEG problem; using its solutions to infer the underlying localization of the neural activity, however intuitively appealing, has no solid theoretical basis. The practice of handpicking or excluding certain solution components based on previous neurophysiological studies can also lead to biasing the results to favor uncertain brain functioning theories; this can be especially a problem when assessing the cortical activity for less well-known tasks, such as imaginary movements. From a purely practical point of view, difficulties will be likely when customizing the BCI interfaces to different patients. Inter-subject variations might impose the need for the manual sorting of the ICA components; thus bringing the risk of introducing arbitrariness and additional errors. We chose to avoid such mistake.

In the present work, we have chosen to objectively analyze the source signals obtained from the ICA algorithm. For this purpose, separating matrices were obtained for each of the movements performed. These matrices were used to estimate to which of the source signal sets a given output vector $x$ was best match. In this way, for each movement type, the set of sources signals $\tilde{s}(k)$ became the features themselves. The types of features used were the same as the ones used to characterize the different frequency bands. (See features 1-40 in Table I).

\section{Feature Selection from DBI indices}

The selection of the features defined previously was based on the Davies-Bouldin Index (DBI) [11]. Refer to [2] for details on how the indices were calculated in this study. The DBI decreases as class separation increases.

\section{Classification}

The five best features, as ranked according to their DBIs (i.e., the estimated class separation), were used for the classification task. In order to compare the effectiveness of the different kinds of features extracted, the classification task was performed independently for the time/frequency and ICA features. In addition to this, a combination of the best features from the two types was tested as well.

Two-thirds of the overall feature data set were taken for training the classifiers; the remaining third was assigned for testing. In order to avoid biasing the results, this process was 
done three times starting with randomized classifiers and data sets. The performance of the classifiers was then averaged over the three resulting feature sets. Five different kinds of classifiers were used: (1) Linear discriminant analysis (LDA), (2) quadratic discriminant analysis (QDA), (3) multilayer perceptron, trained by back-propagation (NNBP), (4) multilayer perceptron, trained by the LevenbergMarquardt rule (NN-LM), and (5) radial basis function neural network (RBFNN).

\section{RESULTS}

\section{A. Left/Right Wrist Movement Classification}

The different kinds of left and right wrist movements were bundled together to estimate our ability to solve this simpler problem. See Table II and Figure 1 for a typical DBI plot corresponding to ICA components. The features are color-coded (darker indicates better clustering); notice how some of the ICA components stand out over the others. See Figure 2 for the corresponding ICA component scalp maps with the lowest-average DBI. Note that whereas components 14 and 16 correspond to activity expected from physiological studies and probably would have been handpicked in other studies, components 12 and 6 provide additional features that could have been overlooked easily.

The five overall best features for every subject (named here $\mathrm{A}, \mathrm{B}, \mathrm{C}$, and D) and movement type (executed or imaginary) are presented in Tables III and IV for time/frequency band and ICA analyses respectively.

Finally, the overall classification failure rates are presented in Table $\mathrm{V}$, tasks for which the success rate was over $70 \%$ are highlighted.

\section{B. Flex/Extend/Pronate/Supinate Classification}

The methodology above unable to yield a success rate over $70 \%$ in any subclass case. The average classification success for the flex/extend/pronate/supinate subclasses were $35 \%, 34 \%, 35 \%$, and $32 \%$, respectively. These values are only marginally better than chance, which would give a $25 \%$ probability of correct classification just by guessing.

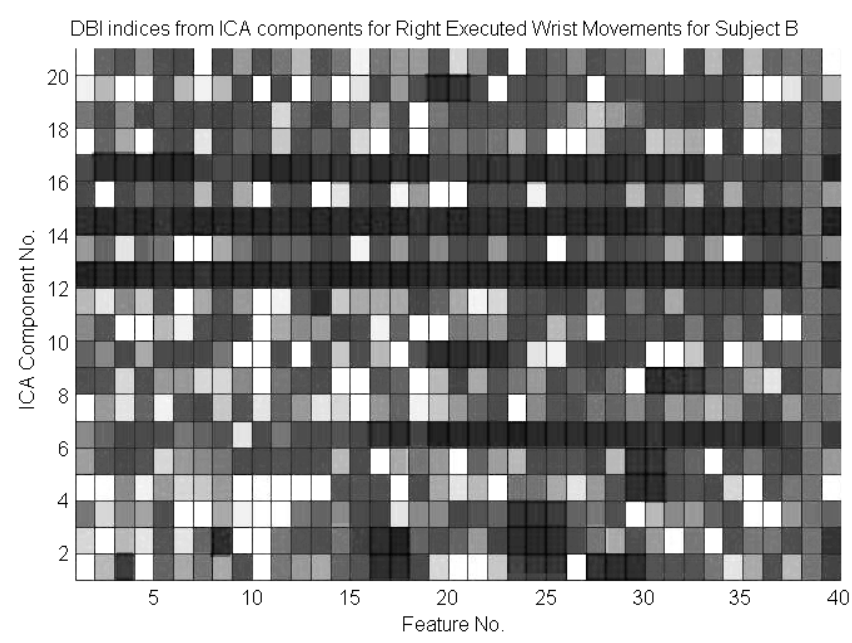

Fig 1 - ICA-based DBI values for subject B. Darker colors indicate smaller DBIs and thus better class separation.
TABLE II

CHANNEL NUMBERS AND LOCATION
\begin{tabular}{|ll|ll|ll|}
\hline Ch & Loc. & Ch & Loc. & Ch. & Loc. \\
1 & Fp1 & 8 & T3 & 15 & Pz \\
2 & Fp2 & 9 & C3 & 16 & P4 \\
3 & F7 & 10 & Cz & 17 & T6 \\
4 & F3 & 11 & C4 & 18 & O1 \\
5 & Fz & 12 & T4 & 19 & O2 \\
6 & F4 & 13 & T5 & 20 & Fpz \\
7 & F8 & 14 & P3 & 21 & Oz \\
\hline
\end{tabular}

ICA Components with Lowest Avg. DBI Subject B I Right-hand wrist executed movements

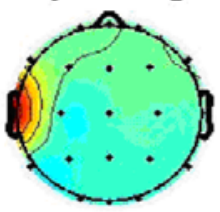

12

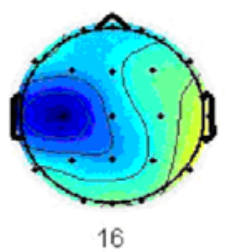

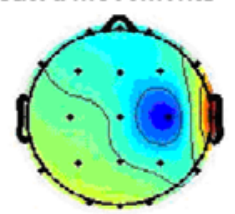

14

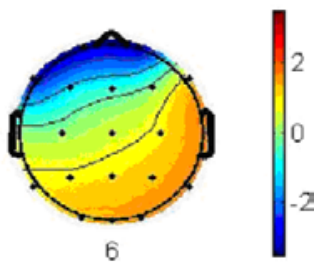

Fig 2- Example of ICA components with the lowest average DBI.

\section{DISCUSSION}

The results indicate that except for the executed movements of subject $\mathrm{A}$, the classifiers were able to yield acceptable left/right classification success rates, ranging from $71 \%$ to $96 \%$. Moreover, the ICA-based classification over-performed the time/frequency band one with a $77 \%$ versus $64 \%$ overall success rate. The application of the Wilcoxon's test to the success rate data also rejected the null hypothesis at the 0.05 level. These results indicate a solid performance, especially if we considered that no effort was devoted to removing any specific artifacts of any kind other than the $50 \mathrm{~Hz}$ mains noise.

In order to improve the quality of the results, new features, better-suited to capture the nature of the alpha and beta bands, will be needed. Note that, for example, for subjects $\mathrm{A}$ and $\mathrm{C}$ most of the features with low DBIs belonged to the beta band. Another analysis that merits further research is the application of the ICA algorithm to each of the alpha, beta, and gamma bands independently. Finally, in order to improve the results for the flex/extend classification tasks it seems likely that the introduction of source localization techniques will be required.

\section{CONCLUSION}

The systematic use of the ICA technique for feature extraction was demonstrated. This technique was then implemented to correctly classify left and right wrist 
movements for either executed or imaginary tasks. Finally, it was shown that the ICA-based approach provided superior performance to that of an approach based solely on frequency band features.

TABLE III

FREQ. BAND TIME-BASED FIVE BEST FEATURES (CHANNEL/FEATURE)

\begin{tabular}{|l|l|l|l|l|l|}
\hline Index rank & 1st & 2nd & 3rd & 4th & 5th \\
\hline $\mathrm{A} /$ Exec & $3 / 120$ & $20 / 120$ & $4 / 120$ & $8 / 89$ & $5 / 10$ \\
\hline $\mathrm{A} / \mathrm{Imag}$ & $3 / 120$ & $8 / 120$ & $9 / 120$ & $13 / 120$ & $4 / 120$ \\
\hline $\mathrm{B} /$ Exec & $1 / 39$ & $14 / 120$ & $20 / 39$ & $2 / 39$ & $3 / 39$ \\
\hline $\mathrm{B} / \mathrm{Imag}$ & $17 / 119$ & $11 / 119$ & $3 / 159$ & $1 / 159$ & $2 / 159$ \\
\hline $\mathrm{C} /$ Exec & $8 / 120$ & $9 / 120$ & $13 / 120$ & $14 / 120$ & $6 / 24$ \\
\hline $\mathrm{C} / \mathrm{Imag}$ & $8 / 113$ & $9 / 115$ & $2 / 39$ & $1 / 39$ & $11 / 112$ \\
\hline $\mathrm{D} /$ Exec & $9 / 80$ & $11 / 80$ & $17 / 79$ & $16 / 79$ & $12 / 80$ \\
\hline $\mathrm{D} / \mathrm{Imag}$ & $11 / 40$ & $17 / 40$ & $16 / 39$ & $1 / 39$ & $19 / 39$ \\
\hline
\end{tabular}

TABLE IV

ICA SOURCES TIME-BASED BEST FIVE FEATURES (LEFT or RIGHT/COMPONENT NO./FEATURE)

\begin{tabular}{|l|l|l|l|l|l|}
\hline Index rank & 1 st & 2nd & 3rd & 4th & 5th \\
\hline $\mathrm{A} /$ Exec & $\mathrm{L} / 10 / 40$ & $\mathrm{R} / 5 / 40$ & $\mathrm{~L} / 5 / 18$ & $\mathrm{~L} / 3 / 40$ & $\mathrm{R} / 8 / 40$ \\
\hline $\mathrm{A} / \mathrm{Imag}$ & $\mathrm{R} / 11 / 40$ & $\mathrm{~L} / 12 / 40$ & $\mathrm{~L} / 15 / 40$ & $\mathrm{R} / 9 / 40$ & $\mathrm{R} / 14 / 40$ \\
\hline $\mathrm{B} /$ Exec & $\mathrm{L} / 1 / 39$ & $\mathrm{~L} / 16 / 40$ & $\mathrm{R} / 14 / 40$ & $\mathrm{~L} / 11 / 39$ & $\mathrm{R} / 12 / 40$ \\
\hline $\mathrm{B} / \mathrm{Imag}$ & $\mathrm{R} / 17 / 40$ & $\mathrm{R} / 13 / 40$ & $\mathrm{~L} / 11 / 40$ & $\mathrm{R} / 9 / 40$ & $\mathrm{~L} / 2 / 39$ \\
\hline $\mathrm{C} /$ Exec & $\mathrm{R} / 5 / 40$ & $\mathrm{~L} / 16 / 40$ & $\mathrm{~L} / 12 / 40$ & $\mathrm{~L} / 10 / 40$ & $\mathrm{~L} / 17 / 40$ \\
\hline $\mathrm{C} /$ Imag & $\mathrm{R} / 11 / 28$ & $\mathrm{~L} / 11 / 28$ & $\mathrm{R} / 19 / 40$ & $\mathrm{R} / 1 / 39$ & $\mathrm{~L} / 14 / 32$ \\
\hline $\mathrm{D} /$ Exec & $\mathrm{L} / 20 / 40$ & $\mathrm{R} / 17 / 40$ & $\mathrm{~L} / 7 / 40$ & $\mathrm{R} / 18 / 14$ & $\mathrm{~L} / 8 / 40$ \\
\hline
\end{tabular}

\section{ACKNOWLEDGEMENTS}

This work has been sponsored by the University of Essex's RPF Fund and by the EPSRC (Grant GR/T09903/01).

\section{REFERENCES}

[1] J. R. Wolpaw, N. Birbaumer, D.J. McFarland, G. Pfurtscheller, and T.M. Vaughan, "Brain-computer interfaces for communication and control," vol. 113, pp. 767-791, 2002.

[2] F. Sepulveda, M. Meckes, and B.A. Conway, "Cluster Separation Index Suggests Usefulness of Non-Motor EEG Channels in Detecting Wrist Movement Direction Intention," Proceedings of the 2004 IEEE Conference on Cybernetics and Intelligent Systems, Singapore, paper FM6. December, 2004.

[3] M. Pregenzer, G. Pfurtscheller, "Frequency Component Selection for an EEG-based Brain to Computer Interface," IEEE Transactions on Rehabilitation Engineering, Vol. 7, No. 4, December 1999.

[4] T. Wang, J. Deng, and B. He, "Classifying EEG-based Motor Imagery Tasks by Means of Time-Frequency Synthesized Spatial Patterns," Clinical Neurophysiology, 115, pp. 2744-2753, 2004.

[5] G. Pfurtscheller, C. Neuper, A. Schlogl, and K. Lugger, "Separability of EEG Signals Recorded During Right and Left Motor Imagery Using Adaptive Autoregressive Parameters," IEEE Transactions on Rehabilitation Engineering, Vol. 6, No. 3, September 1998.

[6] T.-P. Jung, S. Makeig, M.J. McKeown, A.J. Bell, T.-W. Lee, and T.J. Sejnowski, "Imaging Brain Dynamics Using Independent Component Analysis," Proceedings of the IEEE, Vol. 89, No. 7, July 2001.

[7] R. Vigario, J. Sarela, V. Jousmaki, M. Hamalainen, and E.Oja, "Independent Component Approach to the analysis of EEG and MEG Recordings," IEEE Transactions on Biomedical Engineering, Vol. 47, No. 5, May 2000.
[8] A. Delorme, and S. Makeig, "EEGLAB: An Open Source Toolbox for Analysis of Single-Trial EEG Dynamics," Journal of Neuroscience Methods, 134:9-21, 2004.

[9] A. Hyvarinen, and E. Oja, "Independent Component Analysis: Algorithms and Applications," Neural Networks, 13(4-5):411-430, 2000.

[10] A.J. Bell, and T.J. Sejnowski, "An information maximization approach to blind separation and blind deconvolution," Neural Computation, Vol. 7, pp.1129-59, 1995.

[11] J.C. Bezdek, and N. R. Pal, "Some New Indexes of Cluster Validity," IEEE Transactions on Systems, Man, and Cybernetics, Vol. 28(3), pp.301-315, 1998.

TABLE V

LEFT/RIGHT CLASSIFICATION ERROR RATES (TRAINING ERROR/TESTING ERROR)

\begin{tabular}{|l|l|l|l|l|l|}
\hline A/Exec & LDA & QDA & NN-BP & NN-LM & RBFNN \\
\hline Time & $0.38 / 0.39$ & $0.39 / 0.46$ & $0.35 / 0.42$ & $0.29 / 0.45$ & $0.29 / 0.48$ \\
\hline ICA & $0.35 / 0.38$ & $0.34 / 0.39$ & $0.32 / 0.38$ & $0.25 / 0.43$ & $0.27 / 0.42$ \\
\hline Combo & $0.38 / 0.39$ & $0.39 / 0.46$ & $0.32 / 0.45$ & $0.31 / 0.48$ & $0.29 / 0.47$ \\
\hline
\end{tabular}

\begin{tabular}{|l|l|l|l|l|l|}
\hline A/Imag & LDA & QDA & NN-BP & NN-LM & RBFNN \\
\hline Time & $0.31 / 0.40$ & $0.34 / 0.43$ & $0.260 / 0.49$ & $0.20 / 0.46$ & $0.24 / 0.49$ \\
\hline ICA & $0.15 / 0.26$ & $0.21 / 0.22$ & $0.14 / 0.23$ & $0.06 / 0.26$ & $0.09 / 0.24$ \\
\hline Combo & $0.17 / 0.26$ & $0.21 / 0.21$ & $0.13 / 0.23$ & $0.10 / 0.19$ & $0.11 / 0.23$ \\
\hline
\end{tabular}

\begin{tabular}{|l|l|l|l|l|l|}
\hline B/Exec & LDA & QDA & NN-BP & NN-LM & RBFNN \\
\hline Time & $0.24 / 0.37$ & $0.19 / 0.29$ & $0.18 / 0.30$ & $0.09 / 0.26$ & $0.12 / 0.26$ \\
\hline ICA & $0.04 / 0.05$ & $0.05 / 0.05$ & $0.01 / 0.04$ & $0.01 / 0.10$ & $0.02 / 0.14$ \\
\hline Combo & $0.22 / 0.35$ & $0.19 / 0.26$ & $0.08 / 0.21$ & $0.04 / 0.19$ & $0.09 / 0.25$ \\
\hline
\end{tabular}

\begin{tabular}{|l|l|l|l|l|l|}
\hline B/Imag & LDA & QDA & NN-BP & NN-LM & RBFNN \\
\hline Time & $0.29 / 043$ & $0.32 / 0.49$ & $0.22 / 0.38$ & $0.14 / 0.44$ & $0.19 / 0.53$ \\
\hline ICA & $0.16 / 0.25$ & $0.23 / 0.23$ & $0.15 / 0.24$ & $0.09 / 0.21$ & $0.11 / 0.24$ \\
\hline Combo & $0.20 / 0.29$ & $0.24 / 0.28$ & $0.18 / 0.28$ & $0.10 / 0.24$ & $0.14 / 0.29$ \\
\hline
\end{tabular}

\begin{tabular}{|l|l|l|l|l|l|}
\hline C/Exec & LDA & QDA & NN-BP & NN-LM & RBFNN \\
\hline Time & $0.13 / 0.15$ & $0.11 / 0.15$ & $0.09 / 0.15$ & $0.06 / 0.16$ & $0.10 / 0.19$ \\
\hline ICA & $0.12 / 0.14$ & $0.09 / 0.11$ & $0.10 / 0.12$ & $0.18 / 0.29$ & $0.07 / 0.14$ \\
\hline Combo & $0.12 / 0.13$ & $0.09 / 0.11$ & $0.09 / 0.11$ & $0.06 / 0.12$ & $0.08 / 0.17$ \\
\hline
\end{tabular}

\begin{tabular}{|l|l|l|l|l|l|}
\hline C/Imag & LDA & QDA & NN-BP & NN-LM & RBFNN \\
\hline Time & $0.36 / 0.28$ & $0.40 / 0.38$ & $0.23 / 0.28$ & $0.22 / 0.29$ & $0.24 / 0.33$ \\
\hline ICA & $0.27 / 0.29$ & $0.31 / 0.29$ & $0.26 / 0.30$ & $0.15 / 0.25$ & $0.15 / 0.28$ \\
\hline Combo & $0.32 / 0.32$ & $0.39 / 0.42$ & $0.26 / 0.35$ & $0.19 / 0.31$ & $0.20 / 0.39$ \\
\hline
\end{tabular}

\begin{tabular}{|l|l|l|l|l|l|}
\hline D/Exec & LDA & QDA & NN-BP & NN-LM & RBFNN \\
\hline Time & $0.43 / 0.46$ & $0.42 / 0.47$ & $0.38 / 0.47$ & $0.30 / 0.40$ & $0.26 / 0.42$ \\
\hline ICA & $0.10 / 0.11$ & $0.13 / 0.15$ & $0.07 / 0.10$ & $0.04 / 0.11$ & $0.07 / 0.10$ \\
\hline Combo & $0.10 / 0.10$ & $0.13 / 0.14$ & $0.06 / 0.08$ & $0.04 / 0.09$ & $0.09 / 0.19$ \\
\hline
\end{tabular}

\begin{tabular}{|l|l|l|l|l|l|}
\hline D/Imag & LDA & QDA & NN-BP & NN-LM & RBFNN \\
\hline Time & $0.35 / 0.39$ & $0.29 / 0.34$ & $0.23 / 0.32$ & $0.18 / 0.31$ & $0.24 / 0.37$ \\
\hline ICA & $0.29 / 0.30$ & $0.39 / 0.37$ & $0.26 / 0.31$ & $0.18 / 0.31$ & $0.21 / 0.38$ \\
\hline Combo & $0.29 / 0.31$ & $0.29 / 0.32$ & $0.20 / 0.29$ & $0.16 / 0.29$ & $0.22 / 0.37$ \\
\hline
\end{tabular}

